\title{
Managing Non-responders Today and What's on the Horizon
}

\author{
NIRAJ VARMA, MA, MD, PhD, FRCP
}

\author{
Cleveland Clinic, Cleveland, $\mathrm{OH}$
}

\begin{abstract}
An important therapy in the heart failure (HF) population is cardiac resynchronization therapy (CRT). However, a significant minority gain no benefit. Prognosis in this group is generally very poor. Therefore, recognition of and remedial action (if indicated) for non-responders to CRT is of critical value. In practice, this is challenging because one or more of several factors may result in non-response. These are reviewed here and divided into pre-CRT (i.e. appropriate candidate selection), per-CRT (i.e., LV lead deployment) and post-CRT (i.e. programming and monitoring). Newer technologies that may affect degree of response are discussed.
\end{abstract}

KEYWORDS. Cardiac resynchronization therapy, non-CRT therapy, non-responsive.
ISSN 2156-3977 (print) ISSN 2156-3993 (online)

(C) 2016 Innovations in Cardiac Rhythm Management

\section{Introduction}

Cardiac resynchronization therapy (CRT) is an important therapy in the heart failure (HF) population. All trials have shown a durable survival benefit in the long term; ${ }^{1}$ however, trial enrollees have included a large population mix of men, women, patients with and without ischemia, patients with wide and narrow QRS, and those with left bundle branch block (LBBB), right bundle branch block (RBBB), or indeterminate QRS morphologies. As a surrogate for mortality, we have often used reverse remodeling as an index of CRT response. A range of effects on left ventricular (LV) function follows CRT in different individuals. Normalization of LV function improves survival to the level of the general population. ${ }^{2}$ An LV ejection fraction (EF) increase (which correlated with LV end systolic volume changes) of more than $20 \%$ in response to CRT improved survival more than with intermediate (10-20\%) EF improvement. Non-responders-with no sign of reverse remodeling-fared poorly: 4 years after implant, there was a $50 \%$ survival rate, almost similar to those with chronic carcinoma. This is a very poor survival rate. There is a need to identify these patients early and evaluate underlying causes, correct their CRT programming if necessary, or even use

Dr. Varma has received research grants from Medtronic, St. Jude Medical, Biotronik, Sorin and Boston Scientific; speaker for Medtronic, St. Jude Medical and Biotronik; consultant for St. Jude Medical.

Manuscript received February 29, 2016, Final version accepted April 17, 2016.

Address correspondence to: Niraj Varma, MD, PhD, Cleveland Clinic Main Campus Mail Code J2-2, 9500 Euclid Avenue, Cleveland, $\mathrm{OH}$ 44195. E-mail: varman@ccf.org
non-CRT therapy for them. Therefore identifying nonresponders is an important task (Figure 1).

An institutional study published in 2009 by investigators from the Cleveland $\mathrm{Clinic}^{3}$ identified reasons for nonresponse, and many of these reasons still exist today. As shown in Figure 2, suboptimal AV timing was dominant. Other reasons included arrhythmias, which correlate with a reduction in biventricular pacing burden, and decrease survival. We have advanced a great deal in our understanding of suboptimal LV lead position. Little attention is paid to mechanical dyssynchrony today, and an underlying narrow QRS $<120 \mathrm{~ms}$ is no longer an indication for CRT. Another reason for non-response is compliance issues. We can address these reasons for non-response categorized according to pre-CRT, peri-CRT, and post-CRT.

\section{Pre-CRT: candidate selection}

The class I recommendations for CRT now include LBBB and QRS duration $>150 \mathrm{~ms}$. LBBB with a QRS duration of $120-149 \mathrm{~ms}$ is a class IIa indication. For those with non-LBBB patterns, there is a class IIa indication when the QRS duration $>150 \mathrm{~ms}$. These guidelines were built on addressing QRS morphology without attention to QRS duration or QRS duration without attention to QRS morphology. This is an important distinction, because, for instance, we have not examined LBBB with a QRS duration of 120-149 ms in great detail or non-LBBB with varying QRS durations. Morphology as well as duration may matter in single individuals.

Study results have shown that, overall, patients who have RBBB derive no benefit from CRT. For those with non-LBBB and non-RBBB patterns (intraventricular 


\section{Durability of the survival effect of cardiac resynchronization therapy by level of left ventricular functional improvement: Fate of "nonresponders"}

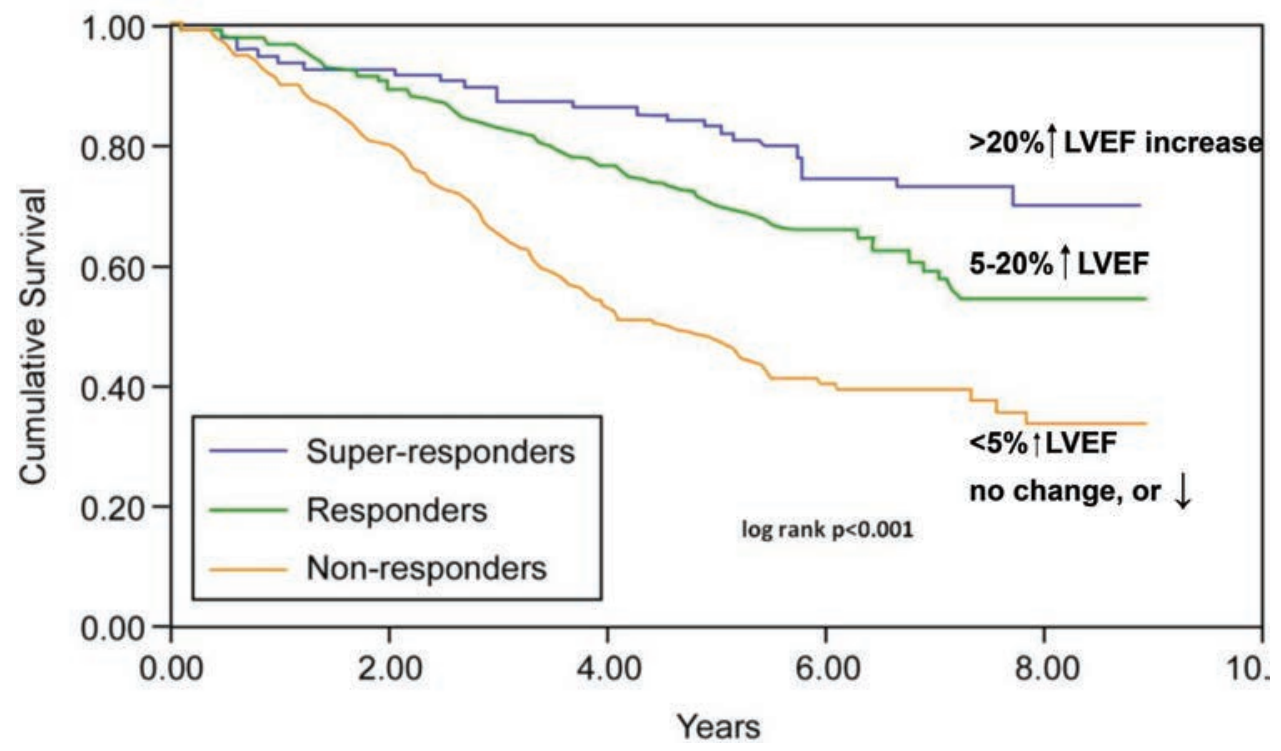

Figure 1: Durability of the survival effect of cardiac resynchronization therapy by level of left ventricular functional improvement: Fate of "non-responders". Reprinted from Heart Rhythm, 11/3, John Rickard, MD, MPH, Alan Cheng, MD, FHRS, David Spragg, MD, FHRS, et al, Durability of the survival effect of cardiac resynchronization therapy by level of left ventricular functional improvement: Fate of "nonresponders", Pages 412-416, 2014, with permission from Elsevier.

conduction defects (IVCD)), CRT may even be harmful. ${ }^{4}$ Within these groups, however, there may be subsets of patients who will benefit. Among those with RBBB, LV activation delay might be masked by the surface QRS. Similarly, with IVCD, there may be coinciding right and left activation delays, but the QRS does not disclose this. Therefore we must look beyond QRS to other methodologies, such as biventricular (BiV) electrical mapping, to identify the area of late activation, direct the lead in that location and program accordingly, and potentially gain CRT benefit.

Patients with LBBB represent a population most likely to respond, but we dichotomize them by a QRS duration of $150 \mathrm{~ms}$. Studies in a patient population with nonischemic cardiomyopathy (NICM) and LBBB have shown that CRT response has high probability for those patients with QRS $>150$ ms. The probability curve ascends from $120 \mathrm{~ms}$ to $150 \mathrm{~ms}$ in QRS duration. It peaks at approximately $150 \mathrm{~ms}$, plateaus, and then dips again. However, QRS duration $>150 \mathrm{~ms}$ does not guarantee $100 \%$ response. There are still non-responders within this group of patients. Among the patient population with QRS duration $<150 \mathrm{~ms}$, at least half of the patients have a potential for response, so we have a responsibility to identify non-responders within the LBBB population. In fact, responders with LBBB compared with non-responders displayed no difference in QRS duration, i.e. this metric was insufficient to discriminate between responders and non-responders to CRT ${ }^{4,5}$ (Figure 2).

This observation indicates that further refinements in patient selection are required. Some address refined interpretations of the 12-lead electrocardiogram (ECG). According to the Strauss criteria, rapid intrinsic conduction in V1, V2, and V3 and then broad QRS complexes, which are notched in contiguous leads V5, V6, and/or I and aVL specifies an ECG pattern within LBBB that is likely to respond to CRT. If we apply electrocardiographic imaging (ECGI) technology, which depicts electrical activation in greater detail, we find that even patients with LBBB have a wide variety of LV conduction patterns. The area of late activation can vary within the $\mathrm{LV}$, and the patterns of LV activation can vary. There is evidence of patterns of LV activation that are particularly responsive to $\mathrm{BiV}$ pacing. This is an aspect that requires investigation in order to address non-responders.

Beyond QRS morphology and duration, other nonelectrical baseline factors may affect CRT response. For instance, sex is a modulator. In a population of patients with LBBB and non-ischemic cardiomyopathy, men had a low frequency of response when QRS duration was $<150 \mathrm{~ms}$. The response in women, on the other hand, peaked at a QRS duration of approximately $135 \mathrm{~ms}$, maintained that peak, and then dipped when QRS duration exceeded $170 \mathrm{~ms}$. These data from a single institution ${ }^{5}$ were 


\section{NICM +LBBB: Probability of CRT Response According to QRS Duration and Gender}
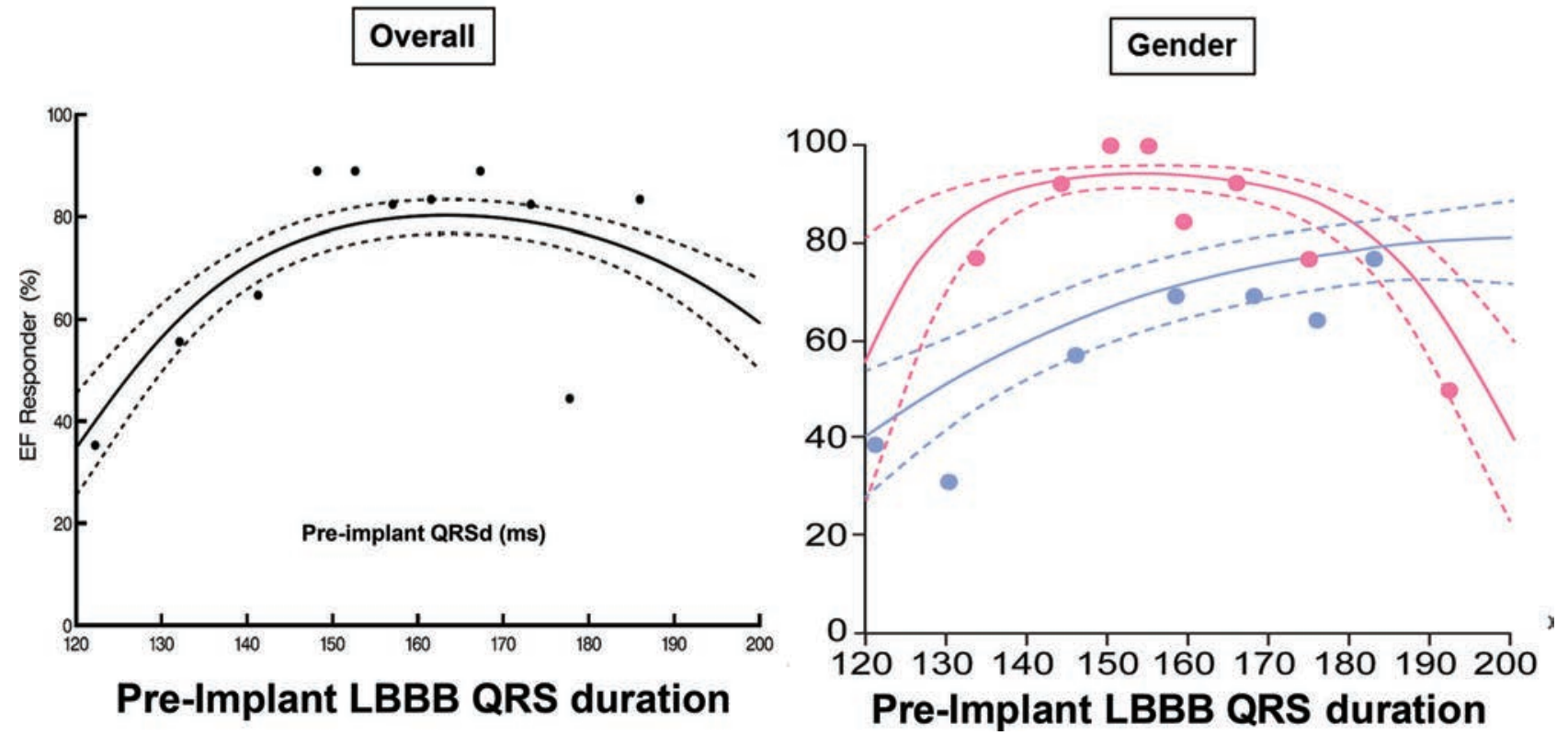

Figure 2: Probability of CRT response according to QRSd as a continuous function. Parametric model: multi-variable logistic regression shown with the corresponding 68\% confidence limits. (A): Overall and (B): Gender-specific plot. Shapes were confirmed by semi-and non-parametric modeling. Reprinted from Heart Rhythm, 11/7, Niraj Varma, MA, DM, Mahesh Manne, MD, Dat Nguyen, DO, Jiayan He, ScD, Mark Niebauer, MD, PhD, Patrick Tchou, MD, Probability and magnitude of response to cardiac resynchronization therapy according to QRS duration and gender in nonischemic cardiomyopathy and LBBB, Pages 1139-1147, 2014, with permission from Elsevier.

subsequently confirmed by results from collected trial data. The data suggest that with QRS duration $<150 \mathrm{~ms}$ and LBBB, CRT should be offered to female patients and a need for modification of the guidelines.

Other comorbidities include ischemia/scar and renal dysfunction. In comparison to the implantable cardioverter defibrillator population, the effect of CRT does not appear to be significantly blunted by underlying renal dysfunction. Pulmonary hypertension and remodeling (LA volume, LV volume, and mitral regurgitation) are significant contributors to CRT response. In order to assess comorbidities, the Multicenter Automatic Defibrillator Implantation Trial with Cardiac Resynchronization Therapy (MADIT-CRT) ${ }^{6}$ evaluated 31 variables and identified seven that might affect future CRT response: female sex, NICM, LBBB, QRS duration > $150 \mathrm{~ms}$, prior HF hospitalization, end diastolic volume, and left atrial volume. In fact, patients with the lowest scores with these seven variables demonstrated no future CRT effect. Hence, a propensity score may be important for patient selection. One item that has not been addressed in scoring indices is the distribution and volume of scar. Greater scar in the
LV correlates with poorer response, because there is less myocardium to recruit for contractile function. ${ }^{7,8}$ The relationship of scar to lead position is important. Pacing into an area of scar reduces CRT effect significantly. ${ }^{9}$ This is not restricted simply to ischemic cardiomyopathy; the effect also prevails among patients with NICM with mid-wall scar. Leyva et al. ${ }^{10}$ conducted a study on LV mid-wall fibrosis as a predicator of mortality and morbidity after CRT and found that the outcome for patients with dilated cardiomyopathy and significant scar was slightly worse than that for patients with general ischemic cardiomyopathy. The response was best for patients with no mid-wall fibrosis. Thus, the presence of scar is significant and must be integrated into candidate selection procedures.

\section{Peri-CRT: LV pacing lead position}

Non-apical lead placement is preferred (and is a class IIa indication in the European Society of Cardiology guidelines). However, where to site the lead non-apically remains less clear. Recommendations for lead position include avoiding posterolateral scar and targeting the site 
of maximal electrical delay. A $\mathrm{qLV}>95 \mathrm{~ms}$ seems to consistently identify patients who will respond versus those who will not be affected by CRT. Data from several studies suggest that the site of maximal mechanical delay is also a determinant of response. The morphology and duration of the paced QRS may be important. Regarding multisite and endocardial pacing strategies, there has been a significant advance in the way that we deliver leads. The multipolar lead provides not only stability and ability to program around phrenic nerve stimulation but also ability to affect future CRT response. In a comparison of distal bipole and proximal bipole, Rajamani et al. ${ }^{11}$ found that the proximal bipole resulted in a qLV measurement of $>95 \mathrm{~ms}$ in $>80 \%$ of patients. Having this span of electrodes and the ability to choose our pacing vectors gives us the opportunity to reach for sites of later activation. In a study of the relationship between $\mathrm{qLV}$ and hemodynamics, $\mathrm{dP} / \mathrm{dt}$ was assessed acutely in a patient population undergoing CRT. ${ }^{12}$ The relationship between $\mathrm{qLV}$ and hemodynamics was almost linear, so the ability to reach for the latest site of electrical activation had significant effect on CRT response in terms of hemodynamics. This is dependent on the availability of coronary sinus tributaries in which to deliver the lead. When they are not available, we must resort to other means.

One option is to use more than one LV lead. Leclercq et al. $^{13}$ conducted studies with multisite LV pacing, while Betts et al. ${ }^{14}$ studied LV endocardial pacing with a transventricular approach. Other previous approaches have been across the atrial septum, but implanting a lead into the LV under these conditions poses significant hazards. However, pacing the endocardium is potentially desirable because LV activation is swifter and LV activation time is reduced.

One interesting new technology involves an LV pellet, which may be delivered retrogradely via the aorta to a site in the LV. A product from EBR Systems transmits ultrasound energy to the $L V$, and this energy is transduced into a pacing output. In the SELECT-LV study, Reddy et al. ${ }^{15}$ applied this technology to conventional CRT failures (non-responders). The preliminary data showed EF improvement at 6 months with the use of this device. This is potentially a promising technique

\section{LV pacing Lead Position: \\ Electrical Effects at Differing Locations}

\author{
Intrinsic \\ Conduction \\ LBBB
}
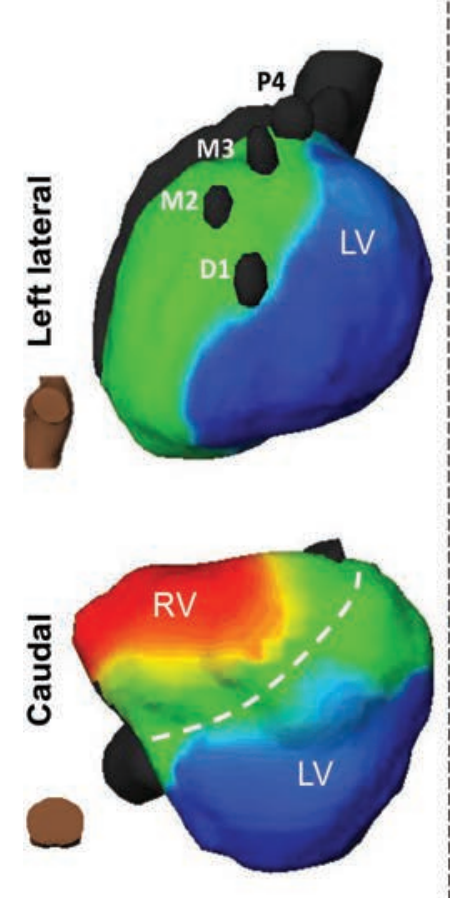

LV Pacing

Quartet Configuration

D1-RV Coil

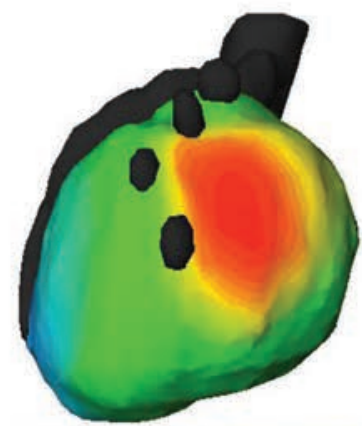

$0 \mathrm{~ms}$

M2- RV Coil
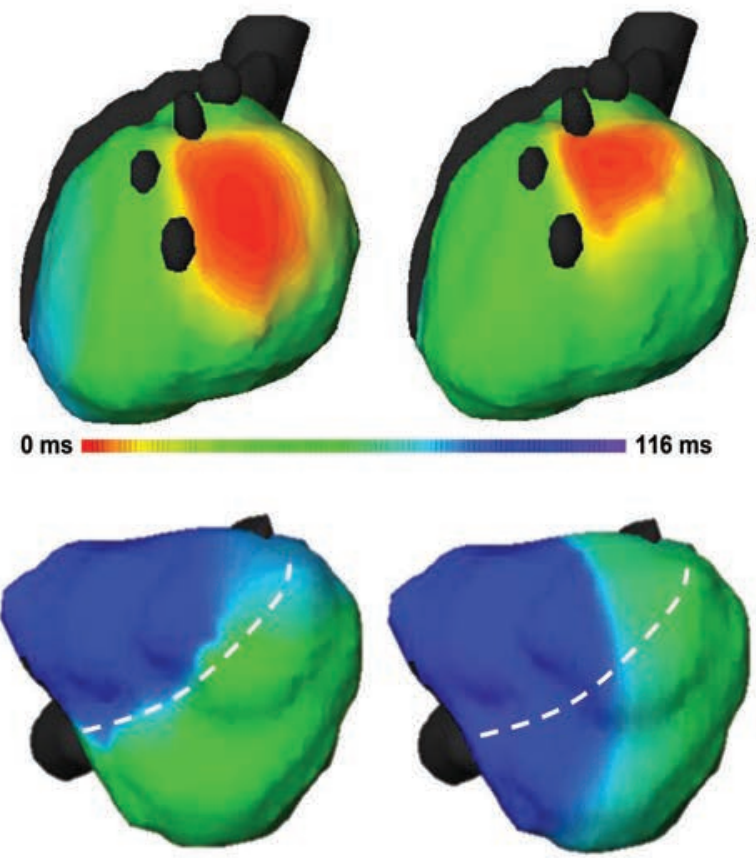

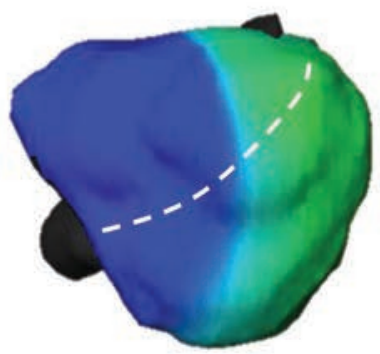

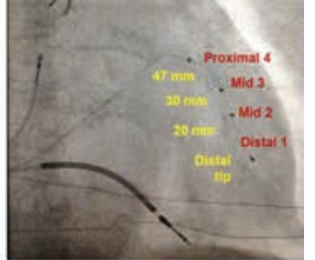

M3-RV Coil
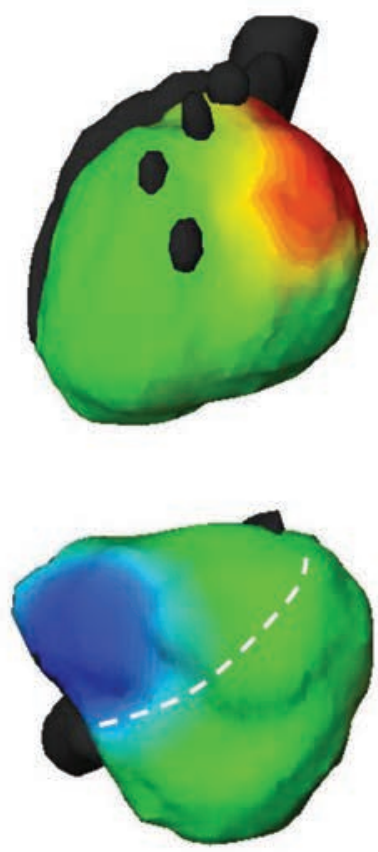

Figure 3: Left ventricular pacing lead position: electrical. Reprinted from Journal of Electrocardiology, 47/1, Niraj Varma, MA, DM, FRCP, Variegated left ventricular electrical activation in response to a novel quadripolar electrode: Visualization by noninvasive electrocardiographic imaging, Pages 66-74, 2014, with permission from Elsevier. 
for achieving LV endocardial pacing, particularly when epicardial pacing via the coronary sinus tributaries is unavailable or ineffective.

We are still confronted with the same challenges in determining which part of the LV to pace. A hemodynamics study from Derval et al. ${ }^{16}$ assessed endocardial pacing sites as well as epicardial pacing sites. There was a wide scatter between best and worst. However, in any single individual, stimulation from the best LV site was twice as good as pacing the worst LV site. The site was widely distributed. The authors concluded that the practice of a fixed single site in the lateral wall will not always capture the hemodynamically best site. This requires individualization. The same applies with electrical activation of the ventricle. In one study, utilization of different biventricular pacing vectors with a quadripolar lead in one individual resulted in highly variable electrical actiovatin sequences when studied with high definition non invasive biventricular mapping (Varma ${ }^{17}$ ) (Figure 3). One that resulted in the best electrical resynchronization also was associated with the narrowest QRS on the surface ECG. Thus, the availability of different pacing vectors is very useful. It can span a larger area of myocardium and give us the ability to pace more hemodynamically and electrically beneficial sites.

\section{Post-CRT: programming and monitoring}

The premise of CRT is that the fusion between the left and right excitation wavefronts correlates with the highest increase in LV contractility. ${ }^{18,19}$ This requires a combination of $\mathrm{AV}$ interval and $\mathrm{VV}$ interval perhaps manifest in the surface QRS. ECG fusion correlates with the best volumetric reverse modeling. ${ }^{20}$ QRS wideners tend to worsen. ${ }^{21}$ However, in trial conditions, the paced QRS does not display significant fusion in almost $50 \%$ of patients, suggesting that we do not pay enough attention to programming after implant.

With echo-guided programming, the principles are that cardiac cycle timing affects hemodynamics and $\mathrm{E}$ and $\mathrm{A}$ waves characterize LV filling, which is a critical part to LV systole. AV optimization uses coordination of the atria and ventricles, maximizes LV filling time, increases cardiac output, and facilitates ventricular synchrony. However, results with $\mathrm{AV}$ optimization have not shown any benefit over out-of-the-box settings. This technique may be of benefit in some individual cases of nonresponse. Optimization of $\mathrm{AV}$ intervals and $\mathrm{VV}$ intervals to reduce ventricular dyssnychrony has also not shown significant effect.

Recent data suggest that electrical programming might be important. We can look at the ECG and change AV and VV intervals to produce the narrowest QRS. In a single-site study from Tamborero et al. ${ }^{22}$ patients randomized to ECG optimization versus echo-guided optimization had better outcomes. Electrical fusion was more predictive of future CRT response. Automatic device-based programming has also been used. With the SMART AV system $^{23}$ and the FREEDOM trial, ${ }^{24}$ investigators examined electrical activation based on interventricular and $\mathrm{AV}$ intervals assessed by the device itself. The two trials did not show benefit, possibly because they were not optimized frequently enough. During diurnal variations, $\mathrm{AV}$ intervals change, and perhaps the need for AV timing and VV timing also change. This is also a limitation to echo-based optimization. However, the Adaptive CRT algorithm self-regulates and reassesses timing on a minute-by-minute interval. The data from that trial have been promising. One analysis from the main trial showed that patients with a higher percentage of synchronized LV pacing in the Adaptive CRT arm had a lower rate of death and HF hospitalizations than echooptimized patients. ${ }^{25}$ This suggests that there is merit in electrical optimization and also in maintaining that optimization and changing it according to needs during the day.

QRS duration may be important. With a quadripolar lead, we have the ability to simultaneously pace different vectors on this lead platform. Zanon and colleagues ${ }^{12}$ showed that QRS duration was significantly abbreviated by MultiPoint ${ }^{\mathrm{TM}}$ Pacing (MPP) (St. Jude Medical, Sylmar, $\mathrm{CA}$ ) compared with standard $\mathrm{BiV}$ pacing. In fact, compared with BiV pacing at any LV site, MultiPoint ${ }^{\text {TM }}$ Pacing (MPP) (St. Jude Medical, Sylmar, CA) yielded a small but consistent contractility increase, which was correlated with greater QRS narrowing, again emphasizing that electrical resynchronization is an important component of increasing response.

Structural heart disease may be a determinant of non-response. Mitral regurgitation is prevalent in CRT recipients. Its severity in the general HF population correlates with adverse ventricular remodeling and outcomes. Bursi et al. ${ }^{26}$ showed that reduction of mitral regurgitation after CRT correlated with an improvement in survival. In contrast, a deterioration in mitral regurgitation correlated with poorer survival. Auricchio et $\mathrm{al}^{27}$ treated 51 severely symptomatic CRT nonresponders with MitraClip (Abbott Laboratories, Abbott Park, IL). Over 12 months, $70 \%$ of patients improved and LV reverse remodeling occurred. This suggests that mitral regurgitation is a significant factor contributing to nonresponse that must be addressed and corrected.

Post-procedural monitoring is very important. Altman et al. ${ }^{28}$ showed improvement in survival with increased intensity of follow-up, multidisciplinary care, and HF evaluation. Schmidt et al. ${ }^{29}$ up-titrated conventional medical therapy after CRT implant and found that improved survival correlated with higher doses of neurohormonal blockers and lower diuretic doses. These results remind us of the importance of adjustment of conventional drug therapy.

Regular follow-up addresses potentially asymptomatic conditions that may cause HF decompensation and deterioration in the future. We can intervene early to prevent the sequence of events that requires higher intensity of follow-up. We know that atrial fibrillation and loss of $\mathrm{BiV}$ pacing correlate with reduced survival, ${ }^{30}$ but in the current era, devices equipped with remote monitoring will inform us of these events. An implanted device will automatically link with telecommunication networks and provide notification 


\section{Post-procedural: Improved Survival with RM}
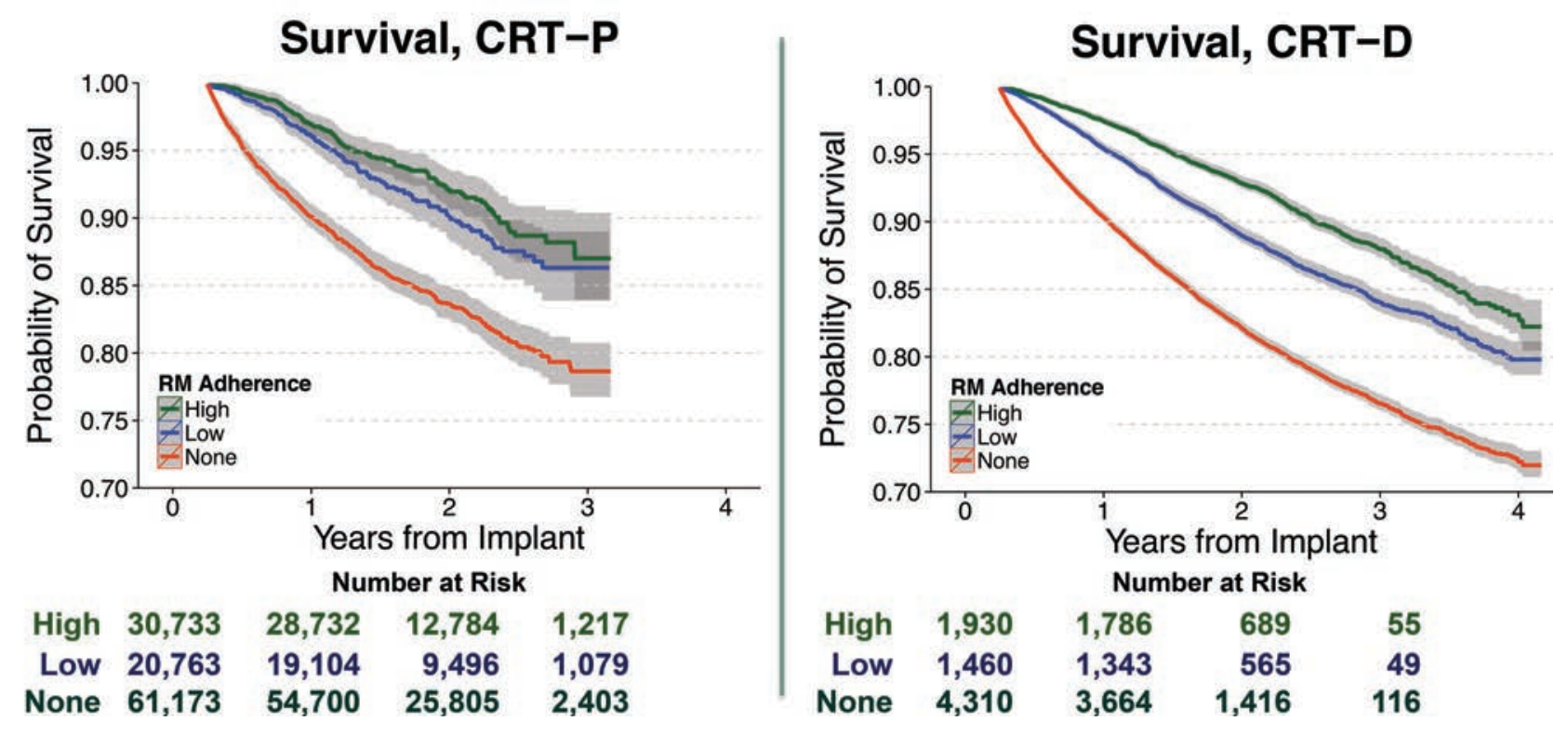

Figure 4: Post-procedural: improved survival with remote monitoring (RM). Reprinted from Journal of the American College of Cardiology, 65/24, Niraj Varma, MD, PhD, Jonathan P. Piccini, MD, MHSc, Jeffery Snell, BA, Avi Fischer, MD, Nirav Dalal, MS, Suneet Mittal, MD, The Relationship Between Level of Adherence to Automatic Wireless Remote Monitoring and Survival in Pacemaker and Defibrillator Patients, Pages 2601-2610, 2015, with permission from Elsevier.

of the advent of atrial fibrillation or loss of BiV pacing or PVC counts. Several other parameters are also available. This information would be automatically relayed within $24-48 \mathrm{~h}$ by the device, regardless of patient symptoms. In a study of patients with CRT-P (pacemaker) and CRT-D (defibrillator), survival was associated with increased adherence to remote monitoring, and survival was reduced in patients who did not maintain consistent follow-up ${ }^{31}$ (Figure 4). The INTIME randomized trial showed a similar survival benefit using remote monitoring with daily transmissions, compared to conventional care. These results point to the importance of follow-up intensity of automatic remote monitoring.

\section{Conclusion}

CRT effect and, therefore, non-response, depend on multiple factors. The magnitude of benefit depends on a variety of underlying conditions. Those with narrow QRS and non-LBBB are very unlikely to gain benefit. Atrial fibrillation may reduce positive effect. Men with ischemic cardiomyopathy have a relatively modest response. Patients with wide QRS and LBBB and women with NICM have the highest probability of response. Selection, electrical substrate, and attention to comorbidities are important. LV lead type, location, pacing effects, and programming are also important. Post-implant monitoring with early reaction to changes in either device function with reprogramming or disease status, for instance, with atrial fibrillation are very important and may affect survival. Attention to one or more of these factors may heighten CRT effect with associated survival benefit. This has not been addressed prospectively in any trials to date, but the ADVANCE CRT registry is evaluating the important patient population of non-responders to CRT.

\section{References}

1. Cleland JG, Abraham WT, Linde C, et al. An individual patient meta-analysis of five randomized trials assessing the effects of cardiac resynchronization therapy on morbidity and mortality in patients with symptomatic heart failure. Eur Heart J. 2013;34(46):3547-3556.

2. Rickard J, Cheng A, Spragg D, et al. Durability of the survival effect of cardiac resynchronization therapy by level of left ventricular functional improvement: fate of "nonresponders". Heart Rhythm. 2014;11(3):412-416.

3. Mullens W, Grimm RA, Verga $T$, et al. Insights from a cardiac resynchronization optimization clinic as part of a heart failure disease management program. J Am Coll Cardiol. 2009;53(9):765-773.

4. Zareba W, Klein H, Cygankiewicz I, et al. MADIT-CRT Investigators. Effectiveness of Cardiac Resynchronization Therapy by QRS Morphology in the Multicenter 
Automatic Defibrillator Implantation Trial-Cardiac Resynchronization Therapy (MADIT-CRT). Circulation. 2011;123(10):1061-1072.

5. Varma N, Manne M, Nguyen D, He J, Niebauer M, Tchou P. Probability and magnitude of response to cardiac resynchronization therapy according to QRS duration and gender in nonischemic cardiomyopathy and LBBB. Heart Rhythm. 2014;11(7):1139-1147.

6. Goldenberg I, Moss AJ, Hall WJ, et al. Predictors of response to cardiac resynchronization therapy in the Multicenter Automatic Defibrillator Implantation Trial with Cardiac Resynchronization Therapy (MADIT-CRT). Circulation. 2011;124(14):1527-1536.

7. Chalil S, Stegemann B, Muhyaldeen SA, et al. Effect of posterolateral left ventricular scar on mortality and morbidity following cardiac resynchronization therapy. Pacing Clin Electrophysiol. 2007;30(10):1201-1209.

8. Ypenburg C, Schalij MJ, Bleeker GB, et al. Impact of viability and scar tissue on response to cardiac resynchronization therapy in ischaemic heart failure patients. Eur Heart J. 2007;28(1):33-41.

9. Leyva F, Foley PW, Chalil S, et al. Cardiac resynchronization therapy guided by late gadolinium-enhancement cardiovascular magnetic resonance. J Cardiovasc Magn Reson. 2011;13:29.

10. Leyva F, Taylor RJ, Foley PW, et al. Left ventricular midwall fibrosis as a predictor of mortality and morbidity after cardiac resynchronization therapy in patients with nonischemic cardiomyopathy. J Am Coll Cardiol. 2012; 60(17):1659-1667.

11. Rajamani K, Wisnoskey B, Varma N. The FOURWARD study; variations in LV electrical delay using quadripolar leads and implications for CRT [abstract P005-187] Heart Rhythm J. 2014;11(5):S553-S591.

12. Zanon F, Baracca E, Pastore G, et al. Multipoint pacing by a left ventricular quadripolar lead improves the acute hemodynamic response to CRT compared with conventional biventricular pacing at any site. Heart Rhythm. 2015;12(5):975-981.

13. Leclercq C, Gadler F, Kranig W, et al. TRIP-HF (Triple Resynchronization In Paced Heart Failure Patients) Study Group. A randomized comparison of triple-site versus dual-site ventricular stimulation in patients with congestive heart failure. J Am Coll Cardiol. 2008;51(15):1455-1462.

14. Betts T, Gamble JH, Khiani R, Bashir Y, Rajappan K. Development of a technique for left ventricular endocardial pacing via puncture of the interventricular septum. Circ Arrhythm Electrophysiol. 2014;7(1):17-22.

15. Reddy VY, Neuzil P, Riahi S, et al. Wireless LV Endocardial Stimulation for CRT: Primary Results of the Safety And Performance Of Electrodes Implanted In The Left Ventricle (SELECT-LV) Study. Paper presented at: 37th Annual Meeting of the Heart Rhythm Society (HRS) for Late Breaking Trials 1-05; May 5, 2016; San Francisco, CA.

16. Derval N, Steendijk P, Gula LJ, et al. Optimizing hemodynamics in heart failure patients by systematic screening of left ventricular pacing sites: the lateral left ventricular wall and the coronary sinus are rarely the best sites. J Am Coll Cardiol. 2010;55(6):566-575.

17. Varma N. Variegated left ventricular electrical activation in response to a novel quadripolar electrode: visualization by non-invasive electrocardiographic imaging. J Electrocardiol. 2014;47(1):66-74.

18. van Gelder BM, Bracke FA, Meijer A, Pijls NH. The hemodynamic effect of intrinsic conduction during left ventricular pacing as compared to biventricular pacing. J Am Coll Cardiol. 2005;46(12):2305-2310.
19. Verbeek XA, Auricchio A, Yu Y, et al. Tailoring cardiac resynchronization therapy using interventricular asynchrony. Validation of a simple model. Am J Physiol Heart Circ Physiol. 2006;290(3):H968-77.

20. Sweeney MO, van Bommel RJ, Schalij MJ, Borleffs CJ, Hellkamp AS, Bax JJ. Analysis of ventricular activation using surface electrocardiography to predict left ventricular reverse volumetric remodeling during cardiac resynchronization therapy. Circulation. 2010;121(5): 626-634.

21. Rickard J, Cheng A, Spragg D, et al. QRS narrowing is associated with reverse remodeling in patients with chronic right ventricular pacing upgraded to cardiac resynchronization therapy. Heart Rhythm. 2013;10(1):55-60.

22. Tamborero D, Vidal B, Tolosana JM, et al. Electrocardiographic versus echocardiographic optimization of the interventricular pacing delay in patients undergoing cardiac resynchronization therapy. J Cardiovasc Electrophysiol. 2011;22(10):1129-1134.

23. Ellenbogen KA, Gold MR, Meyer TE, et al. Primary results from the SmartDelay determined AV optimization: a comparison to other AV delay methods used in cardiac resynchronization therapy (SMART-AV) trial: a randomized trial comparing empirical, echocardiography-guided, and algorithmic atrioventricular delay programming in cardiac resynchronization therapy. Circulation. 2010; 122(25):2660-2668.

24. Abraham WT, Gras D, Yu CM, Guzzo L, Gupta MS, FREEDOM Steering Committee. Rationale and design of a randomized clinical trial to assess the safety and efficacy of frequent optimization of cardiac resynchronization therapy: the Frequent Optimization Study Using the QuickOpt Method (FREEDOM) trial. Am Heart J. 2010;159(6):944-948.e1.

25. Martin DO, Lemke B, Birnie D, et al. Adaptive CRT Study Investigators. Investigation of a novel algorithm for synchronized left-ventricular pacing and ambulatory optimization of cardiac resynchronization therapy: results of the adaptive CRT trial. Heart Rhythm. 2012;9(11):1807-1814.

26. Bursi F, Barbieri A, Grigioni F, et al. Prognostic implications of functional mitral regurgitation according to the severity of the underlying chronic heart failure: a long-term outcome study. Eur J Heart Fail. 2010;12(4):382-388.

27. Auricchio A, Schillinger W, Meyer S, et al. PERMIT-CARE Investigators. Correction of mitral regurgitation in nonresponders to cardiac resynchronization therapy by MitraClip improves symptoms and promotes reverse remodeling. J Am Coll Cardiol. 2011;58(21):2183-2189.

28. Altman RK, Parks KA, Schlett CL, et al. Multidisciplinary care of patients receiving cardiac resynchronization therapy is associated with improved clinical outcomes. Eur Heart J. 2012;33(17):2181-2188.

29. Schmidt S, Hürlimann D, Starck CT, et al. Treatment with higher dosages of heart failure medication is associated with improved outcome following cardiac resynchronization therapy. Eur Heart J. 2014;35(16):1051-1060.

30. Hayes DL, Boehmer JP, Day JD, et al. Cardiac resynchronization therapy and the relationship of percent biventricular pacing to symptoms and survival. Heart Rhythm. 2011;8(9):1469-1475.

31. Varma N, Piccini JP, Snell J, Fischer A, Dalal N, Mittal S. The Relationship Between Level of Adherence to Automatic Wireless Remote Monitoring and Survival in Pacemaker and Defibrillator Patients. J Am Coll Cardiol. 2015;65(24):2601-2610. 